\title{
Properties of mapping induced by fornix damages: Learning and memorizing the radial maze task
}

\author{
MARTINE AMMASSARI-TEULE \\ Laboratoire de Physiologie Nerveuse, C.N.R.S., Gif-sur-Yvette, France \\ and Istituto di Psicobiologia e Psicofarmacologia del C.N.R., Rome, Italy \\ and \\ CATHERINE MAHO \\ Laboratoire de Physiologie Nerveuse, C.N.R.S., Gif-sur-Yvette, France
}

\begin{abstract}
A correlational analysis of learning scores and degrees of divergence of the patterns of choice was performed in fornix-damaged, sham-operated, and control rats during the acquisition stage of the radial maze task. The results show that dorsal fornix sections, which initially reduce choice accuracy, induce the parallel adoption of weakly divergent patterns of choice. The positive correlation observed during the course of training between these two dependent variables suggests that fornix-damaged animals learn the task on the basis of specific gathering and processing of information: initial choice of few proximal paths with progressive increases in the number of visited paths under the control of reinforcement determines a correlative increase in the degrees of divergence of the patterns of choice. This observation indicates that mapping in lesioned animals occurs through the progressive introduction of new spatial elements in an initial sectorial exploratory system, whereas sham-operated and control animals immediately process more information on the total configuration of the apparatus. This particular gathering and processing of spatial information could determine specific reference and working memory processes in lesioned animals.
\end{abstract}

In the past few years, the radial maze task has been used to study spatial memory in relation to the hippocampal function (Jarrard, 1980; O'Keefe \& Nadel, 1978; Olton, Becker, \& Handelmann, 1979; Walker \& Olton, 1979; Winocur, 1982). However, from this extensive literature, several controversial issues have arisen concerning spatial memory characteristics and the underlying mechanisms. From a behavioral viewpoint, discrepancies have been observed regarding the persistence of information over time (Beatty \& Shavalia, 1980a; Burešova \& Bureš, 1982 ), the resistance of working memory to various sources of interference (Beatty \& Shavalia, 1980b; Maki, Brokofsky, \& Berg, 1979; Markowska, Burešova, \& Bureš, 1983), and the identification of the stimulus controlling the performance (Burešova \& Bureš, 1981; Kraemer, Gilbert, \& Innis, 1983; Olton \& Collison, 1979; Suzuki, Augerinos, \& Black, 1980; Winocur, 1982; Zoladek \& Roberts, 1978). From a neural viewpoint, although the integrity of the hippocampal system and of its connections seems necessary to learn and perform the radial maze task, it is still unclear if this structure supports a

The authors are indebted to Gerard Dutrieux, who wrote the program for calculating the degrees of divergence of the patterns of choice, and to Gerard Le Floch for his technical assistance. Correspondence may be directed to M. Ammassari-Teule at Istituto di Psicobiologia e Psicofarmacologia, Via Reno 1, 00198 Rome, Italy. specific processing of spatial information for cognitive mapping (O'Keefe \& Nadel, 1978) or more general functions such as patterning of responses (Moore, 1979; Solomon, 1980), working memory (Olton \& Feustle, 1981), reactivity to novelty (Jones \& Smith, 1980), sensitivity to environmental modifications (Winocur, 1980), contextual retrieval (Hirsh, 1974), general processing and utilization of information (Jarrard \& Elmes, 1982), and, finally, computational and representational activities (Schmajuk, 1984).

However, since the solving of the radial maze task depends on the integration of various processes-orientation, motivation, mapping, memory of places, organizations of run sequences - the functional involvement of the hippocampal system in this specific spatial task could be evaluated by focusing on the organization of the responses and, in particular, on the mapping operations that occur in lesioned and control animals during the course of acquisition.

Few investigations have systematically analyzed the acquisition process of the radial maze, since most experiments have been performed using the following procedure: training the subjects until asymptotic performance and then examining, in retention, the modifications of spatial memory in relation to lesions, interference, or drug treatments. Furthermore, while much attention has been devoted to choice accuracy, there has been little consider- 
ation of the strategies used by the subjects to learn the task.

Indeed, when the patterns of choice have been analyzed, correct sequences have generally been considered and classified in only two categories: clockwise or counterclockwise (corresponding to the run of adjacent arms within a trial) versus spatial strategies (Olton, Collison, \& Werz, 1977; Watts, Stevens, \& Robinson, 1981; Yoerg \& Kamil, 1982) or random versus stable patterns (Harley, 1979). However, it must be pointed out that in an eightarm maze 8 ! $(40,320)$ correct sequences are available to solve the task, among which only 16 correspond to clockwise and counterclockwise patterns. Thus, the remaining sequences cannot be considered to be in one homogeneous class of responses, inasmuch as they express various degrees of the structuring of the spatial information that is used in running the radial maze, which will probably lead to different representations of the apparatus configuration. In the same way, the various structures of the incorrect patterns could also induce different forms of mapping, which could determine a different organization of reference and working memory processes.

The present experiment was undertaken to examine the relationships between mapping operations and the functioning of spatial memory in fornix-damaged, shamoperated, and control rats. For this purpose, a correlational analysis of the quantitative (choice accuracy) and qualitative (patterns of choice) aspects of radial maze performance was performed in order to describe, in parallel, the evolution of both dependent variables during the course of acquisition.

Subsequently, a long-term examination of spatial memory was also performed in sequential and discrete trials in order to relate the functioning of reference and working memory processes to the properties of the response systems built up during acquisition.

\section{METHOD}

\section{Subjects}

The subjects were 24 male rats (Sprague-Dawley) that weighed about $180 \mathrm{~g}$ when purchased from the IFFA CREDO rearing center. They were individually housed in wire-mesh cages with ad-lib food and water. Two days before pretraining began, the subjects were progressively food deprived to bring their weights to $85 \%$ of their initial levels.

\section{Apparatus}

The apparatus was a maze consisting of eight identical paths $(60 \times 12 \mathrm{~cm})$ radiating from a starting platform. Identical food cups were placed at the end of each path. The maze was elevated $60 \mathrm{~cm}$ above the floor, uniformly illuminated by a ceiling light, and maintained in a constant orientation during the experiment. Numerous objects present in the experimental room provided extramaze cues.

\section{Pretraining}

For 3 consecutive days, each subject was placed on the central platform and then, for $5 \mathrm{~min}$, allowed to explore the maze, in which a large amount of food had been placed.

\section{Surgery}

On the 4th day, the surgery was performed. Fornix sections were made by introducing a stereotaxic knife close to the bregma (using the following coordinates: A-P, -0.5; Lat, 0.5; depth, 4.74; Paxinos \& Watson, 1982) and moving the blade according to a predetermined angle $\left(40^{\circ}\right)$ to obtain a section of the dorsal fornix. Sham operations were made in exactly the same way except that the blade was not extracted from the knife. The subjects were left in their home cage for a recovery period of 1 week.

\section{Training}

On the 12 th day, the actual procedure started. For 8 consecutive days, the rats were placed in the maze and allowed to make eight runs, with all the paths having been previously baited. On the 20th day, delays of $5 \mathrm{~min}$ were inserted within the trial between the first four runs and the last four. On the 21st day, the control rats were submitted to fornix sections as previously described. After 1 week, all the animals were placed in the maze for a normal trial. Finally, on the subsequent day, a delay of $20 \mathrm{~min}$ was inserted between the first four runs and the last four runs.

\section{Behavioral Analysis}

In each trial, two dependent variables were recorded for each subject: the number of visited paths and the sequence of runs.

As previously mentioned, the number of possible correct sequences of runs in an eight-arm maze is 40,320 . These sequences can be grouped according to their common degree of divergence. This parameter, which was introduced by Lachman and Brown (1957), is obtained as follows: Within a trial, all the transitions between two arms run consecutively are evaluated by counting one unit of divergence when the rat runs two adjacent arms successively; by counting two units of divergence when the rat successively runs two arms separated by one arm; by counting three units of divergence when the rat successively runs two arms separated by two arms; and so on. For the whole sequence of runs displayed within the trial, the units of divergence corresponding to each transition are summed and give the final value, that is, the degree of divergence of the sequence corresponding to one category. For example, for the sequence of runs "1-2-4-5-3-6-7-8," the degree of divergence will be obtained by summing up the following units of divergence: " $1+2+1+2+3+1+1$," which give the number 11 . It must be noted that the degree of divergence of clockwise and counterclockwise strategy is 7 and that 16 different patterns correspond to this category. Since, in this experiment, the number of runs within a trial is constant (eight), it is also possible to calculate the degree of divergence of incorrect patterns. Therefore, for each animal, each daily performance was characterized by two numbers: the number of visited paths and the degree of divergence of the corresponding pattern of choice.

\section{Statistical Analysis}

For each dependent variable, that is, learning scores and degrees of divergence of the strategies, the results recorded over the eight learning trials were statistically evaluated by a one-way ANOVA for repeated measures. Correlations between these two dependent variables were examined by calculating the Bravais Pearson coefficient. Comparisons of scores and degrees of divergence of the strategies in each retention trial were made by performing a KruskallWallis nonparametric analysis of variance because of the small size of the distributions.

\section{RESULTS}

\section{Histology}

At the end of the experiment, lesioned and shamoperated subjects were sacrificed. The brains were fixed 


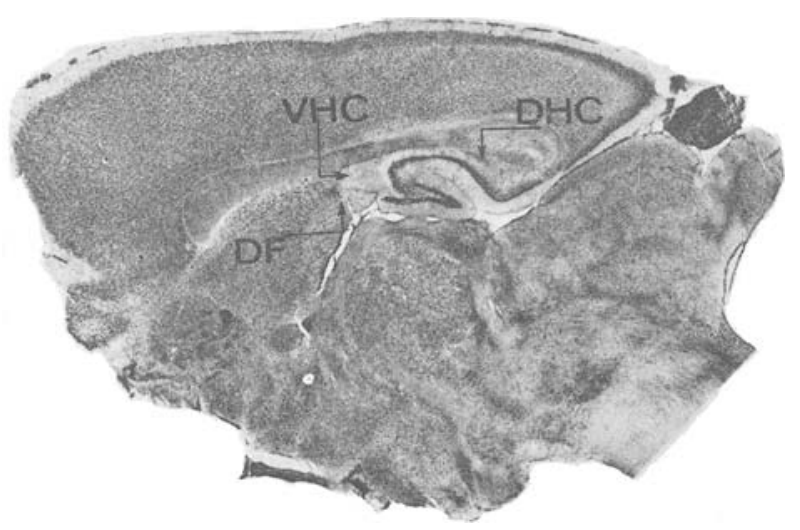

Figure 1. Sagittal section representative of the dorsal fornix section. DHC, dorsal hippocampal commissure; VHC, ventral hippocampal commissure; DF, dorsal fornix.

in Formalin, stained with cresyl violet, and sectioned sagitally. Examination of the tissue revealed clear dorsal fornix sections extended in some cases to the hippocampal commissure (Figure 1).

\section{Behavior}

As can be seen in Figure 2a, which shows for each group the mean number of unrepeated path choices in the first eight trials, choice accuracy was impaired in fornixdamaged rats as compared with sham-operated and control rats. The statistical analysis revealed a significant effect of the experimental treatment $[\mathrm{F}(2,20)=3.59$, $\mathrm{p}<.05]$ and a significant effect of training $[\mathrm{F}(7,140)=$ $8.49, p<.01]$. Subsequent between-group comparisons performed by a Duncan test revealed that the performance of lesioned rats was significantly different from the performance of sham-operated and control rats $(p=.05)$, between which no difference was found.

The same analysis was used to compare the degrees of divergence of the strategies recorded in the three groups. The results showed a significant treatment effect $[F(2,20)=3.94, p<.05]$, indicating that the degrees of divergence of the patterns of choice displayed in the three groups were different, but no effect of the repeated measures or of the interaction was observed. A subsequent Duncan test also revealed that the degrees of divergence recorded in fornix-damaged animals were significantly different from those recorded in sham-operated and control animals $(p<.05)$, between which no difference was found.

Moreover, with reference to Figure $2 b$, it can be seen that although the degrees of divergence displayed by shamoperated and control rats follow the same stable trend throughout the experiment, this parameter is more variable in fornix-damaged rats. Therefore, a correlative analysis of performance and degree of divergence of patterns of choice was performed. A positive correlation was found between choice accuracy and degree of divergence of the strategies in fornix-damaged animals only $(r=.36$, $\mathrm{p}=.01$ ), the lowest learning scores corresponding to the weakest divergent strategies. Finally, it must be noted that the percentage of correct trials was similar in each group (Table 1) and that no clockwise or counterclockwise patterns were displayed over the whole experiment.

When delays of $5 \mathrm{~min}$ were inserted between two strings of runs (Trial 9), no significant differences either in choice accuracy $(\mathrm{H}=1.2$, n.s.) or in strategies were observed $(\mathrm{H}=4.49$, n.s. $)$. In the same way, when the animals were tested in long-term retention-after 1 week without practice-no quantitative $(\mathrm{H}=1.7$, n.s. $)$ or qualitative $(\mathrm{H}=2.47$, n.s. $)$ change in performance occurred (Trial 10). Finally, when delays of 20 min were inserted between two strings of runs (Trial 11), choice accuracy decreased in the same proportion in the three groups but modifications of the strategies occurred only in shamoperated rats. The statistical analysis revealed significant differences among the strategies displayed in the three groups $(H=11.7, p<.01)$. Subsequent between-group comparisons indicated that only sham-operated rats reorganized their strategies in discrete trials, since the animals of this group differ from both the initially and the later-lesioned animals (sham-operated/lesioned$\mathrm{U}=8.5, \mathrm{p}=.06$; lesioned/recently lesioned- $\mathrm{U}=24$, n.s.; sham-operated/recently lesioned- $U=10.5, p=.01$ ).
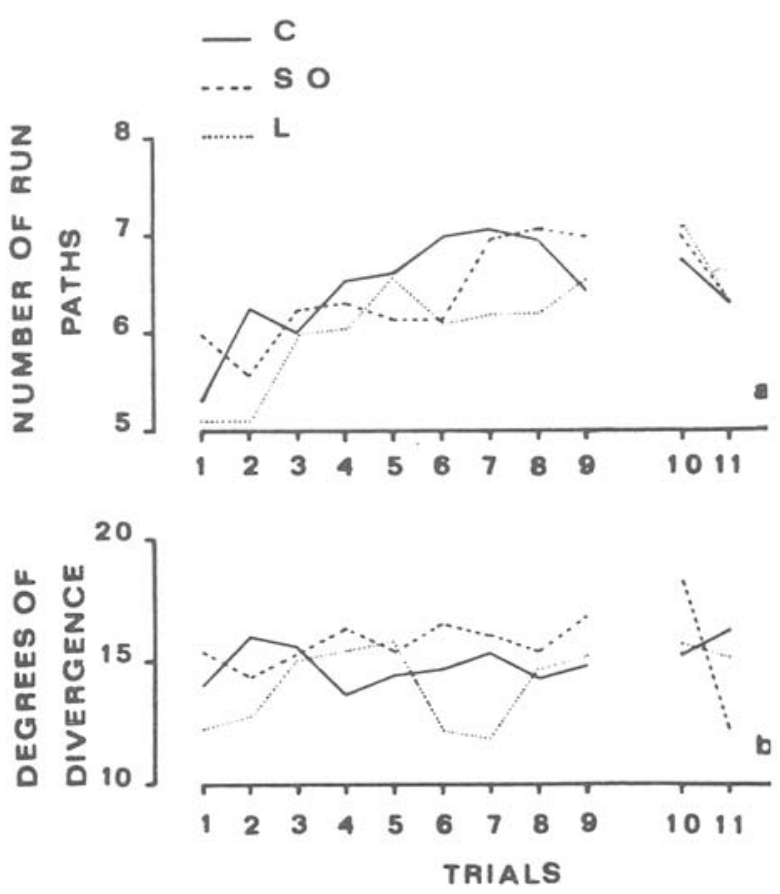

Figure 2. Learning scores (a) and degrees of divergence of the corresponding patterns of choice (b). The learning scores are expressed in mean number of run paths within each trial; the calculation of the degrees of divergence of the patterns of choice is developed in the method section. C, control group to Trial 9, lesioned from Trial 10; SO, sham-operated group; L, lesioned group. 
Table 1

Percentage of Correct Sessions and Their Corresponding Mean Degree of Divergence (With Standard Error in Parentheses) in Each Group During the 8 Days of Training

\begin{tabular}{lcl}
\hline \multicolumn{1}{c}{ Group } & Percentage & $\begin{array}{l}\text { Degrees of } \\
\text { Divergence }\end{array}$ \\
\hline Lesioned & $7.0 \%$ & $15.8(1.0)$ \\
Sham-operated & $12.0 \%$ & $16.0(2.3)$ \\
Control & $8.3 \%$ & $14.8(2.6)$ \\
\hline
\end{tabular}

\section{DISCUSSION}

The present findings confirm previous data concerning the impairment of radial maze performance following fornix damage (Walker \& Olton, 1979). Moreover, they show that fornix sections, which initially reduce choice accuracy, induce a parallel adoption of weakly divergent patterns of exploration during the acquisition stage of the radial maze task. This last result can be viewed as an extention of previous observations that have indicated a response bias in rats submitted to successive choices in T-maze situations after transection of the fornix fibers (Hirsh, Leber, \& Gillman, 1978). In particular, the fact that lesioned animals display a correlative enhancement of choice accuracy and degrees of divergence of their patterns of choice during the course of acquisition suggests that fornix damage induces a specific informationgathering process that is characterized by the progressive introduction of new spatial dimensions in an initial sectorial exploratory system. Conversely, it is clear that mapping in sham-operated and in control animals involves the immediate processing of more information on the general configuration of the apparatus, since these animals display highly divergent and stable patterns of choice right at the beginning of the experiment. Accordingly, these data raise two questions: First, what mechanisms induce fornix-damaged animals to adopt weakly divergent sequences of exploration in learning the radial maze task; and, second, what kinds of constraints will this form of mapping impose on successively tested reference- and working-memory processes?

Although deficits in attentional (Moore \& Stickney, 1982 ) or in working-memory processes (Olton \& Feustle, 1981) could be invoked to explain the low scores recorded in fornix-damaged subjects, it seems unlikely that the restrained explorative tendencies that also characterize the performance of these animals depend on impairments in their discrimination of the paths or in their memory of previous choices. In fact, sequences of runs involving repetitive choices that would be randomly distributed in all arms of the radial maze would better fit with these hypotheses.

Perseverative behaviors, which are frequently reported in hippocampally damaged subjects (Buzsaki, Bors, Nagy, \& Eidelberg, 1982; Kimble \& Kimble, 1965; Oades \& Isaacson, 1978), could also be invoked to explain both the low radial maze scores and the reduced exploration rates observed in our lesioned animals. However, since perseveration seems to depend on the inability to inhibit previously made responses (Kimble \& Kimble, 1965) or to develop normal processes of stimulus satiation (Glanzer, 1953), a more dramatic reduction in the degree of divergence of the patterns of choice would be associated with the development of mere perseverative tendencies.

The specific mapping of the radial maze developed by fornix-damaged subjects reveals, rather, an initial tendency to process a limited quantity of spatial information by exploring, first, only sectors of the apparatus, with these restrained explorative schemas being progressively extended until the entire maze configuration under the control of reinforcement. However, since all the subjects reached a comparable level of performance in long-term retention, our results fully agree with the Hirsh et al. (1978) hypothesis, suggesting that the difference in the way normal and hippocampally damaged animals learn must be viewed as a stylistic difference. As a working hypothesis, we assume that this stylistic difference in processing information during acquisition could determine in each group specific properties of subsequently tested reference- and working-memory processes.

On Trial 9, when short delays were inserted between two strings of runs, choice accuracy and degrees of divergence of the patterns of choice remained similar to those observed in each group on Trial 8. The stable scores observed in lesioned, sham-operated, and control animals could be due to the reduced interference resulting from the very short delay introduced between the first four and the last four runs. It is interesting to note that the slightnot significant-working-memory deficit observed in control animals only supports the Jarrard and Elmes (1982) hypothesis, according to which hippocampally damaged rats would be less sensitive than normal rats to retroactive interference.

The data concerning long-term retention (Trial 10) do not show any between-groups variations of performance or patterns of choice, even in the previous control group now bearing dorsal fornix sections. Finally, on Trial 11, when 20 min were inserted between two strings of runs, choice accuracy decreased in the same proportion in each group, indicating no difference in working-memory processes of lesioned and sham-operated animals. However, the absence of a memory deficit, probably due to the partial transections of the fornix, allows a specific effect of the lesion on patterns of choice to be observed, since very different maze-running strategies are now associated with these similar performances: (1) fornixdamaged animals exhibit a remarkable stability in the degree of divergence in their patterns of choice with respect to those displayed in the previous trials, (2) recently fornix-damaged animals follow the same stable trend, and (3) sham-operated animals significantly reduce the degree of divergence of their patterns of choice.

Thus, when required to perform a slightly modified task that involves substantial time intervals between the fourth and the fifth choices, which place an additional load on working-memory processes, sham-operated rats do reorganize their maze-running strategies to maintain a consistent level of choice accuracy. Conversely, lesioned 
animals perform through the reproduction of rigid patterns of choice (which do not implicate the running of the same paths but do implicate the development of spatially equivalent patterns of exploration) not substantially different from the "motor stratagems" described by Rawlins and Tsaltas (1983) and defined as motor-based systems of instructions mediating the appropriate response not only in sequential, but also in discrete, trials.

In fact, in Figure 2 it can be seen that lesioned animals later display stable degrees of divergence as compared with sham-operated and control animals, but that this stabilization occurs when the animals have reached a consistent level of performance (after Trial 6) and is maintained even in trials involving interrun intervals (Trials 9 and 11).

Taken together, these results indicate that the septohippocampal pathway controls both aspects of spatial memory, that is, choice accuracy and mapping operations. In fact, dorsal fornix sections induce constraints on spatial information gathering, which has been considered as an active process whereby the animals build up their representations of the maze configuration (Ellen \& Weston, 1983). The specific representations stored in reference memory by fornix-damaged subjects could determine a particular functioning of working memory, based upon a limited number of plans (Gray, 1982) that are not highly accurate but resistant to retroactive interference.

\section{REFERENCES}

Beatty, W. W., \& Shavalia, D. A. (1980a). Rat spatial memory: Resistance to retroactive interference at long-term retention intervals. Animal Learning \& Behavior, 8, 550-552.

Beatty, W. W., \& Shavalia, D. A. (1980b). Time course of working memory and effects of anesthetics. Behavioral \& Neural Biology, 28, 454-462.

BuREŠOVA, O., \& BurEš, J. (1981). Role of olfactory cues in the radial maze performance of rats. Behavioural Brain Research, 3, 405-409.

BUREŠova, O., \& BUREš, J. (1982). Capacity of working memory in rats as determined by performance on the radial maze. Behavioural Processes, 7, 63-72..

Buzsaki, G., Bors, L., Nagy, F., \& Eidelberg, E. (1982). Spatial mapping, working memory and the fimbria fornix system. Journal of Comparative \& Physiological Psychology, 96, 26-34.

Ellen, P., \& Weston, S. L. (1983). Problem solving in the rat: Septal lesion effects on habituation and perseverative tendencies. Physiological Psychology, 11, 112-118.

Glanzer, M. (1953). Stimulus satiation: An explanation of spontaneous alternation and related phenomena. Psychological Review, 60. 26-34.

Gray, J. A. (1982). The neurophysiology of anxiety: An enquiry into the function of the septo-hippocampal system. Oxford: Oxford University Press.

HaRLEY, C. (1979). Arm-choice in a sunburst maze: Effects of hippocampectomy in the rat. Physiology \& Behavior, 23, 283-290.

HiRSH, R. (1974). The hippocampus and contextual retrieval of information from memory: A theory. Behavioral Biology, 12, 421-444.

HirSh, R., Leber, B., \& Gillman, K. (1978). Fornix fibers and motivational states as controllers of behavior: A study stimulated by the contextual retrieval theory. Behavioral Biology, 22, 463-478.

JARRARD, L. E. (1980). Selective hippocampal lesions and behavior. Physiological Psychology, 8, 198-206.

JARRARD, L. E., \& ElmEs, D. G. (1982). Role of retroactive interference with spatial memory of normal rats and rats with hippocampal lesions. Journal of Comparative \& Physiological Psychology, 96, 699-711.

JoNES, D. G., \& SMITH, B. J. (1980). The hippocampus and its response to differential environments. Progress in Neurobiology, 15, 19-69.

KimBLE, D. P., \& KimBLE, R. J. (1965). Hippocampectomy and response perseveration in the rat. Journal of Comparative \& Physiological Psychology, 60, 472-476.

Kraemer, P. J., Gilbert, M. E., \& INNIS, N. K. (1983). The influence of cue type and configuration upon radial maze performance in the rat. Animal Learning \& Behavior, 11, 373-380.

LACHMAN, S. J., \& BRown, C. R. (1957). Behavior in a free choice multiple path elimination problem. Journal of Psychology, 13, 101-109.

MAKI, W. S., BrokofsKy, S., \& BERG, B. (1979). Spatial memory in rats: Resistance to retroactive interference. Animal Learning \& Behavior, 7, 25-30.

Markowska, A., Burešova, O., \& Bureš, J. (1983). An attempt to account for controversial estimates of working memory persistence in the radial maze. Behavioral \& Neural Biology, 38, 97-112.

Moore, J. W. (1979). Information processing in space-time by the hippocampus. Physiological Psychology, 7, 224-232.

Moore, J. W., \& STICKNEY, K. J. (1982). Goal tracking in attentional associative networks: Spatial learning and the hippocampus. Physiological Psychology, 10, 202-208.

OADES, R. D., \& ISAACSON, R. L. (1978). The development of foodsearch behavior in rats: The effects of hippocampal damage and haloperidol. Experimental Neurology, 24, 327-337.

O'KeEFE, J., \& NADEL, L. (1978). Hippocampus as a cognitive map. London: Oxford University Press.

Olton, D. S., Becker, J. T., \& Handelmann, G. E. (1979). Hippocampus, space and memory. Behavioral \& Brain Sciences, 2, 313-365.

Olton, D. S., \& Collison, C. (1979). Intramaze cues and "odor trails" fail to direct choice behavior on an elevated maze. Animal Learning \& Behavior, 7, 221-223.

Olton, D. S., Collison, C., \& Werz, M. A. (1977). Spatial memory and radial-arm maze performance of rats. Learning \& Motivation, 8, 289-314.

Olton, D. S., \& Feustle, W. D. (1981). Hippocampal function refined for nonspatial working memory. Experimental Brain Research, 41, 380-389.

Paxinos, G., \& Watson, C. (1982). The rat brain in stereotaxic coordinates. London, Sydney, \& New York: Academic Press.

Rawlins, J. N. P., \& Tsaltas, E. (1983). The hippocampus, time and working memory. Behavioural Brain Research, 10, 233-262.

Schmajuk, N. A. (1984). Psychological theories of hippocampal function. Physiological Psychology, 12, 166-183.

Solomon, P. R. (1980). A time and a place for everything? Temporal processing views of hippocampal function with special reference to attention. Physiological Psychology, 8, 254-261.

Suzuki, S., Augerinos, G., \& BlaCk, A. H. (1980). Stimulus control of spatial behavior on the eight-arm maze. Learning \& Motivation, 11, 1-18.

WAlker, J. A., \& Olton, D. S. (1979). Spatial memory deficits following fimbria-fornix lesions: Independence of time for stimulus processing. Physiology \& Behavior, 23, 11-15.

Watts, J., Stevens, R., \& Robinson, C. (1981). Effects of scopolamine on radial maze performance in rats. Physiology \& Behavior, 26, 845-851.

Winocur, G. (1980). The hippocampus and cue utilization. Physiological Psychology, 8, 280-288.

WinOCUR, G. (1982). Radial-arm-maze behavior by rats with dorsal hippocampal lesions: Effects of cuing. Journal of Comparative \& Physiological Psychology, 96, 155-169.

Yoerg, S. I., \& KAMIL, A. C. (1982). Response strategies in the radial maze: Running around in circles. Animal Learning \& Behavior, 10, 530-534.

ZOLADEK, L., \& ROBERTS, W. A. (1978). The sensory basis of spatial memory in the rat. Animal Learning \& Behavior, 6, 77-81.

(Manuscript received May 25, 1985;

revision accepted for publication December 20, 1985.) 